\title{
A Set Theoretic Approach for Time to Contact Estimation in Dynamic Vision
}

\author{
A. Garulli \\ D. Prattichizzo \\ A. Vicino \\ Dipartimento di Ingegneria dell'Informazione, Università di Siena \\ Via Roma, 56 - 53100 Siena, Italia \\ garulli,prattichizzo,vicino@ing.unisi.it
}

\begin{abstract}
This paper deals with the estimation of the time to contact in dynamic vision problems. A novel approach based on set membership estimation theory is proposed. It allows for the computation of bounds on the time to contact estimates in finite time.
\end{abstract}

\section{Introduction}

One of the main problems in robot visual navigation and in robot manipulation systems is the determination of the time to contact (or time to collision). In mobile robots, the recovery of this parameter is of paramount importance in collision avoidance and in braking. Reference [15] is one of the first papers where vision-based spatio-temporal techniques enable the robotic device to avoid collisions by computing the time to contact (see [7] for a comprehensive bibliography on the topic).

The basic problem consists in computing the time needed for the observer to reach a fixed object, in the hypothesis that the relative velocity along the optical axis is kept constant.

Within the world of dynamic vision, this specific problem appears as a typical 'goal oriented' problem, in the sense that its solution does not require the complete 3-D scene reconstruction, but it can be accomplished in an efficient way by using only a partial solution of the general motion to structure problem. Two main approaches have been proposed in the literature.

The first one [7] is based on differential invariants of the image velocity field (curl, divergence and shear), which are used to characterize the changes in the shape of objects in the scene due to the relative motion between the observer and the scene. Under the hypothesis of constant velocity along the optical axis, the time to contact turns out to be a function of the area enclosed by the object contour and its time derivative. One advantage of this method consists in the fact that the evaluation of the time to contact is performed without tracking point features in the image, i.e. estimating the full image ve-

0-7803-4394-8/98 \$10.00 ( 1998 IEEE locity field, which is an ill conditioned problem. In fact, since the estimates are based on surface integrals along the contours, the method is weakly sensitive to noise measurements. The main drawback of this technique consists in its sensitiveness to partial occlusions of the object.

The second approach is based on the estimation of the optical flow [12]. As such, this method shows good ability in following fast changes in the time to contact, but because it involves estimation of derivatives of the optical flow, it is quite sensitive to measurement noise.

This paper focuses on the differential invariants approach [7] and its aim is twofold. First, a new recursive estimation technique is introduced for evaluating the variables involved in the computation of the time to contact. The new approach relies on an estimation theory developed quite recently in the system identification area of research, where it is known as 'set membership' or 'unknownn but bounded' approach (see e.g. $[16,13,14])$. The basic assumption underlying this theory is that error and noise processes belong to prescribed sets. These sets are generally defined in terms of norms. The objective is to find optimal set estimators and evaluate the corresponding minimal uncertainty intervals for the unknown variables to be estimated.

The second contribution of the paper consists in providing theoretical results which allow for the computation of guaranteed uncertainty intervals for the time to contact estimate in a finite number of steps. This topic is important in all the problems where an explicit evaluation of the accuracy of the estimate of the time to contact is crucial. For example, obstacle avoidance in autonomous navigation of mobile robots fall in this category of problems.

Set membership techniques for the estimation of the time to contact through the optical flow approach are reported in [9] and in [10].

This paper is organized as follows. Section 2 describes the approach based on contour tracking and introduces the set membership approach used to track contours parameterized according to B-splines snakes. Section 3 solves the problem of evaluating nonconservative uncer- 
tainty intervals for the time to contact estimates computed through the technique proposed in Sections 2

\section{Contour tracking}

The linear parameterization of image contours is largely used in computer vision. B-splines interpolation shows attractive features for fitting both open and closed contours (see e.g. [3]). In fact, it is well known that this parameterization leads to numerically well behaved solutions and to efficient algorithms.

A closed B-spline curve of degree $h$ is defined as

$$
\mathcal{S}(u)=\sum_{i=0}^{m-1} \mathbf{V}_{i} B_{i h}(u), \quad u \in\left[u_{\min }, u_{\max }\right],
$$

where $B_{i h}(u)$ are the B-spline base functions of degree $h$ and the coefficients $\mathrm{V}_{i}=\left(X_{i}, Y_{i}\right)$ are the 'control points' of the curve. The base functions satisfy a number of constraints at the knots of the curve, ensuring a given level of regularity of the interpolating curve [1].

The contour tracking problem consists in computing an estimate $\hat{\mathcal{S}}(u)$ of the object contour as a function of the control points $\mathbf{V}_{i}$, evolving dynamically with time. The problem is frequently approached through Kalman filtering theory. This is done by constructing a state space model of the rigid object contour motion. State variables are defined as the coordinates $X_{i}(t), Y_{i}(t)$ of the control points and their respective time derivatives

$$
\begin{array}{ll}
\mathbf{X}=\left[X_{0}, \ldots, X_{m-1}\right]^{\prime}, & \mathbf{Y}=\left[Y_{0}, \ldots, Y_{m-1}\right]^{\prime} \\
\dot{\mathbf{X}}=\left[\dot{X}_{0}, \ldots, \dot{X}_{m-1}\right]^{\prime}, & \dot{\mathbf{Y}}=\left[\dot{Y}_{0}, \ldots, \dot{Y}_{m-1}\right]^{\prime}
\end{array}
$$

Assuming a planar motion with uniform velocity, the contour dynamics equations are given as

$$
\frac{d}{d t}\left[\begin{array}{c}
\mathbf{X} \\
\dot{\mathbf{X}}
\end{array}\right]=\left[\begin{array}{ll}
0 & I \\
0 & 0
\end{array}\right]\left[\begin{array}{c}
\mathbf{X} \\
\dot{\mathbf{X}}
\end{array}\right]+\left[\begin{array}{c}
0 \\
I
\end{array}\right] \mathbf{w}_{x}
$$

where $\mathbf{w}$ represents the process noise accounting for randomly varying forces acting on the object. An equation similar to (2) holds for the state variables $\mathbf{Y}$ and $\dot{\mathbf{Y}}$.

The visual measurement process consists in collecting $L$ measurements of the contour position, by searching along normal rays to the estimated curve and within a search window, whose size is function of the positional variance of the current estimate. It is convenient to express measurements of the system output in terms of the state vectors:

$$
z_{x}(u, t)=\left[\begin{array}{ll}
B(u) & \mathbf{0}
\end{array}\right]\left[\begin{array}{c}
\mathbf{X} \\
\dot{\mathbf{X}}
\end{array}\right]+v_{x}(u, t)
$$

where $v$ is the measurement noise and $B(u)$ is a vector whose components are the B-spline base functions $B_{i h}(u), i=0, \ldots, m-1$. A similar equation holds for measurements $z_{y}(u, t)$ of the second coordinate of the control points.

\subsection{Planar affine transformation of contours}

If the shape of the image is complex, the parameterization through B-splines requires a large number of control points. As a consequence, the dimension of the state space vector built by grouping control points in (1) dramatically increases leading image tracking algorithms to instability [3].

Usually, for many purposes, e.g., the computation of the time to contact, the considered object are rigid bodies, as for instance the area of the rear windscreen of a car in automatic parking applications. In such cases, the control points parameterization would allow the image contour redundant degrees of freedom. In fact, it is known [18] that the projection onto the image plane of a planar rigid object, moving with respect to the camera, can be completely described by just six affine degrees of freedom embodied by a vector $\mathbf{Q}$ defined as

$$
\mathbf{Q}=\left[\begin{array}{llllll}
t_{x} & t_{y} & s r_{11} & s r_{22} & s r_{21} & s r_{12}
\end{array}\right]^{\prime}
$$

where $t_{x}\left(t_{y}\right)$ represents the translation of the contour in the image plane along the $x(y)$ direction, $s$ the scaling factor and $r_{i j}$ the entry $(i, j)$ of the rotation matrix $(\operatorname{det}(R)=1)$.

The $\mathbf{Q}$-parameterization describes the contour relatively to the template [8], i.e., the initial reference estimated contour denoted by control points $(\overline{\mathbf{X}}, \overline{\mathbf{Y}})$. The relationship between the $Q$ vector and the control points of a single captured image is given by

$$
\left[\begin{array}{l}
\mathbf{X} \\
\mathbf{Y}
\end{array}\right]=W \mathbf{Q}+\left[\begin{array}{c}
\overline{\mathbf{X}} \\
\overline{\mathbf{Y}}
\end{array}\right]
$$

where

$$
W=\left[\begin{array}{cccccc}
1 & 0 & \overline{\mathbf{X}} & \mathbf{0} & \mathbf{0} & \overline{\mathbf{Y}} \\
\mathbf{0} & \mathbf{1} & \mathbf{0} & \overline{\mathbf{Y}} & \overline{\mathbf{X}} & \mathbf{0}
\end{array}\right]
$$

From (5) it is easy to check that

$$
\mathbf{Q}=M\left[\left[\begin{array}{l}
\mathbf{X} \\
\mathbf{Y}
\end{array}\right]-\left[\begin{array}{l}
\overline{\mathbf{X}} \\
\overline{\mathbf{Y}}
\end{array}\right]\right]
$$

where $M$ is the $H$-weighted pseudoinverse

$$
M=\left(W^{\prime} H W\right)^{-1} W^{\prime} H .
$$

Matrix $H$ is a metric matrix [4] arising from the normal equations for the problem of least-squares approximation with B-splines.

The 12-dimensional vector of state variables is here defined as $\left[\mathbf{Q}^{\prime} \dot{\mathbf{Q}}^{\prime}\right]^{\prime}$ being $\dot{\mathbf{Q}}$ the time derivative of $\mathbf{Q}$

$$
\dot{\mathbf{Q}}=M\left[\begin{array}{c}
\dot{\mathbf{X}} \\
\dot{\mathbf{Y}}
\end{array}\right]
$$

From (2) and (7) the contour dynamics in the affine state space is easily obtained as

$$
\frac{d}{d t}\left[\begin{array}{l}
\mathbf{Q} \\
\dot{\mathbf{Q}}
\end{array}\right]=\left[\begin{array}{ll}
0 & I \\
0 & 0
\end{array}\right]\left[\begin{array}{l}
\mathbf{Q} \\
\dot{\mathbf{Q}}
\end{array}\right]+\left[\begin{array}{l}
0 \\
I
\end{array}\right] M\left[\begin{array}{l}
\mathbf{w}_{x} \\
\mathbf{w}_{y}
\end{array}\right]
$$


As regards the visual measurements process, equation (3) and its $y$ counterpart turn into

$$
\begin{aligned}
& {\left[\begin{array}{c}
\Delta z_{x}(u, t) \\
\Delta z_{y}(u, t)
\end{array}\right]=\left[\begin{array}{c}
z_{x}(u, t)-\bar{z}_{x}(u) \\
z_{y}(u, t)-\bar{z}_{y}(u)
\end{array}\right]} \\
& \left.=\left[\begin{array}{cc}
B(u) & 0 \\
0 & B(u)
\end{array}\right] W \quad 0\right]\left[\begin{array}{c}
\mathbf{Q} \\
\hline \dot{\mathbf{Q}}
\end{array}\right]+\left[\begin{array}{l}
v_{x}(u, t) \\
v_{y}(u, t)
\end{array}\right]
\end{aligned}
$$

where $\left(\bar{z}_{x}(u), \bar{z}_{y}(u)\right)$ represent the contour of the template $(B(u) \overline{\mathbf{X}}, B(u) \overline{\mathbf{Y}})$.

\subsection{Discrete time dynamics of image contour}

In order to derive a tracking algorithm for the image contour, the discrete-time version of the contour dynamics should be computed. The discrete-time dynamics is derived for the control points approach first and for the affine parameterization after. For both approaches the discrete dynamics and the measurement equation assume the following form

$$
\left\{\begin{array}{l}
\lambda_{k+1}=\mathbf{F} \lambda_{k}+\mathbf{G} \mathbf{w}_{k} \\
\mathbf{y}_{k}=\mathbf{H} \lambda_{k}+\mathbf{v}_{k}
\end{array}\right.
$$

where $\lambda_{k}$ represent the state vector at time $k$ and $\mathbf{w}_{k}$ $\left(\mathbf{v}_{k}\right)$ is the input (measurement) noise.

When the control points parameterization approach is used, the dynamics of the $\mathbf{X}$ and $\mathbf{Y}$ coordinates can be analyzed separately. For the $\mathbf{X}$-coordinate (2) of the image contour, the state vector at time $k$ is $\lambda_{k}=$ $\left[\begin{array}{ll}\mathbf{X}_{k}^{\prime} & \dot{\mathbf{X}}_{k}^{\prime}\end{array}\right]^{\prime}$, while vector $\mathbf{w}_{k}$ represents the process noise $\mathbf{w}_{x, k}$ at time $k$. By discretizing continuous dynamics (2), one gets

$$
\mathbf{F}=\left[\begin{array}{cc}
I_{m} & \delta t I_{m} \\
0_{m} & I_{m}
\end{array}\right] \quad, \quad \mathbf{G}=\left[\begin{array}{c}
\frac{\delta t^{2}}{2} I_{m} \\
\delta t I_{m}
\end{array}\right]
$$

with $\delta t$ denoting the sampling period and $I_{m}$ standing for the $m$-dimensional identity matrix.

The set of visual measurements available at time $k$, $\mathbf{y}_{k}=\left[\begin{array}{lll}z_{x}\left(u_{1}, k\right) \ldots & \ldots & z_{x}\left(u_{L}, k\right)\end{array}\right]^{\prime}$, is a vector containing $L$ measurements of the contour position, $\mathbf{v}_{k}=$ $\left[v_{x}\left(u_{1}, k\right) \ldots v_{x}\left(u_{L}, k\right)\right]^{\prime}$ is the measurement noise vector, and the output matrix in (10) can be written as

$$
\mathbf{H}=\left[\begin{array}{ll}
\overline{\mathbf{H}} & \mathbf{0}
\end{array}\right]
$$

being $\overline{\mathbf{H}}$ a matrix whose components $\bar{H}_{i j}$ are the B-spline base functions $B_{j h}\left(u_{i}\right), i=1, \ldots, L, j=$ $0, \ldots, m-1$.

According to $(8,9)$, the discrete-time version of the contour dynamics for the affine parameterization approach, is simply obtained. The state vector at time $k$ is here $\lambda_{k}^{\prime}=\left[\mathbf{Q}^{\prime} \dot{\mathbf{Q}}^{\prime}\right]^{\prime}$, while process noise vector at time $k$ becomes $\mathbf{w}_{k}=\left[\mathbf{w}_{x, k}^{\prime} \mathbf{w}_{y, k}^{\prime}\right]$. The dynamics and disturbance matrices of (10) are easily derived as

$$
\mathbf{F}=\left[\begin{array}{cc}
I_{6} & \delta t I_{6} \\
0_{6} & I_{6}
\end{array}\right] \quad, \quad \mathbf{G}=\left[\begin{array}{c}
\frac{\delta t^{2}}{2} I_{6} \\
\delta t I_{6}
\end{array}\right] M
$$

The $2 L$ visual measurements available at time $k$ are grouped into vector

$\mathbf{y}_{k}=\left[\Delta z_{x}\left(u_{1}, k\right) \Delta z_{y}\left(u_{1}, k\right) \ldots \Delta z_{x}\left(u_{L}, k\right) \Delta z_{y}\left(u_{L}, k\right)\right]^{\prime}$

and the output matrix in (10) can be written as

$$
\mathbf{H}=[\overline{\mathbf{H}} \mathbf{0}]
$$

being

$$
\overline{\mathbf{H}}=\left[\begin{array}{c}
{\left[\begin{array}{cc}
B\left(u_{1}\right) & 0 \\
0 & B\left(u_{1}\right)
\end{array}\right] W} \\
\\
\vdots \\
{\left[\begin{array}{cc}
B\left(u_{L}\right) & 0 \\
0 & B\left(u_{L}\right)
\end{array}\right] W}
\end{array}\right]
$$

Finally, in the $\mathbf{Q}$-parameterization approach, the measurement noise vector assume the following form

$$
\mathbf{v}_{k}=\left[v_{x}\left(u_{1}, k\right), v_{y}\left(u_{1}, k\right) \ldots v_{x}\left(u_{L}, k\right), v_{y}\left(u_{L}, k\right)\right]^{\prime}
$$

\subsection{Set membership filtering}

Following an alternative approach with respect to the literature, it will be assumed that both process and measurement noises in (10) are unknown-but-bounded $[16,13,14,11]$. This means that the a priori knowledge on the uncertainty affecting the system can be expressed as

$$
\begin{aligned}
\left\|\mathbf{w}_{k}\right\|_{\infty} & \leq \varepsilon_{w} \\
\left\|\mathbf{v}_{k}\right\|_{\infty} & \leq \varepsilon_{v},
\end{aligned}
$$

where $\varepsilon_{w}$ and $\varepsilon_{v}$ are known positive scalars and $\|\mathrm{a}\|_{\infty} \triangleq$ $\sup _{i}\left|a_{i}\right|$. In this setting, the problem of computing a recursive estimate of the state vector is addressed in a set theoretic approach, see e.g. $[2,5,6]$. Assume that the initial state vector $\lambda_{0}$ lies in the bounded set $\mathcal{X}(0 \mid-1)$. Then, denote by $\mathcal{X}(k \mid k)$ and $\mathcal{X}(k+1 \mid k)$ the sets of state vectors $\lambda_{k}$ and, respectively, $\lambda_{k+1}$, which are compatible with all the available information up to time $k$ (namely the noise bounds (15)-(16), the initial condition $\mathcal{X}(0 \mid-1)$ and the measurements $\mathrm{y}_{i}$, for $i=$ $0,1, \ldots, k)$. It is easy to see that the above sets are provided by the following recursion

$$
\begin{aligned}
\mathcal{X}(k \mid k) & =\mathcal{X}(k \mid k-1) \bigcap \mathcal{M}(k) \\
\mathcal{X}(k+1 \mid k) & =\mathbf{F} \mathcal{X}(k \mid k)+\mathbf{G} \mathcal{S}_{\infty}\left(\varepsilon_{w}\right)
\end{aligned}
$$

where $\mathcal{M}(k)=\left\{\lambda_{k}:\left\|\mathbf{y}_{k}-\mathbf{H} \lambda_{k}\right\|_{\infty} \leq \varepsilon_{v}\right\}$ is the set of state vectors compatible with the measurements at time $k$, and $\mathcal{S}_{\infty}\left(\varepsilon_{w}\right)$ is the ball of radius $\varepsilon_{w}$ in the $\infty$-norm. In general, as $k$ increases, the sets $\mathcal{X}(k \mid k)$ and $\mathcal{X}(k+1 \mid k)$ become very complicated and their exact computation requires a large amount of calculations. Therefore, approximation through simple regions like ellipsoids $[2,5]$ is often pursued. In this paper, the approximating regions adopted are parallelotopes. An $n$-dimensional parallelotope is defined as

$$
\mathcal{P}=\left\{\lambda \in \mathbb{R}^{n}: \lambda=\hat{\lambda}+\mathbf{T} \alpha,\|\alpha\|_{\infty} \leq 1\right\}
$$


where $\hat{\lambda}$ is the center of the parallelotope and $\mathbf{T}$ is the $n \times n$ matrix whose column vectors represent the edges of the parallelotope.

Recursive approximation of polytopic regions through parallelotopes has been introduced in [19], and then applied to the state estimation problem in [6], where two sequences of outer approximating parallelotopes $\mathcal{P}(k \mid k)$ and $\mathcal{P}(k+1 \mid k)$ are computed so that they satisfy the inclusions

$$
\begin{aligned}
\mathcal{P}(0 \mid-1) & \supseteq \mathcal{X}(0 \mid-1) \\
\mathcal{P}(k \mid k) & \supseteq \mathcal{P}(k \mid k-1) \bigcap \mathcal{M}(k) \\
\mathcal{P}(k+1 \mid k) & \supseteq \mathbf{F} \mathcal{P}(k \mid k)+\mathbf{G S}_{\infty}\left(\varepsilon_{w}\right)
\end{aligned}
$$

for $k=0,1, \ldots$ In order to reduce the conservativeness introduced by the approximation, minimum volume parallelotopes are considered. The measurements $z_{x}\left(u_{i}, k\right)$ contained in the vector $\mathbf{y}_{k}$ are processed sequentially, so that the approximating boxes can be updated according to the ROBP Algorithm introduced in [6]. Hence, at time $k$, the set theoretic parallelotopic state filter provides the set estimate

$$
\begin{aligned}
\mathcal{P}(k \mid k) & =\left\{\lambda_{k \mid k}:\right. \\
\lambda_{k \mid k} & \left.=\hat{\lambda}_{k \mid k}+\mathbf{T}_{k \mid k} \alpha, \quad\|\alpha\|_{\infty} \leq 1\right\}
\end{aligned}
$$

where $\hat{\lambda}_{k \mid k}$ is the central estimate of the state vector and $\mathbf{T}_{k \mid k}$ defines the uncertainty region associated with the central estimate. The uncertainty intervals relative to each control point coordinate, and corresponding time derivative, can be obtained by computing the minimum volume axis-aligned box containing the parallelotope $\mathcal{P}(k \mid k)$. The following lemma provides the desired outbounding box.

Lemma 1. Consider the paralleotope $\mathcal{P}(k \mid k)$ in (21), and let $\mathbf{T}_{k \mid k}=\left\{t_{i j}\right\}$. Then, the minimum volume axisaligned box containing $\mathcal{P}(k \mid k)$ is given by

$$
\begin{aligned}
\mathcal{B}(k \mid k) & =\left\{\lambda_{k \mid k}:\right. \\
\lambda_{k \mid k} & \left.=\hat{\lambda}_{k \mid k}+\mathbf{D}_{k \mid k} \alpha, \quad\|\alpha\|_{\infty} \leq 1\right\}
\end{aligned}
$$

where $\mathbf{D}_{k \mid k}=\operatorname{diag}\left(d_{1}, \ldots, d_{2 m}\right)$ and $d_{i}=\sum_{j}\left|t_{i j}\right|, i=$ $1, \ldots, 2 m$.

\subsection{Computation of the time to contact}

The time to contact (or time to collision) $\tau$ is defined as the time interval between the present instant and the instant when the observing sensor and the point on the object along the optical axis come to collision, under the hypothesis of uniform relative motion.

Denoting by $A(t)$ the area enclosed by the contour estimated at time $t$, and by $\dot{A}(t)$ its time derivative, it can be shown that the time to contact $\tau$ is given by [7]

$$
\tau=\frac{2 A(t)}{\dot{A}(t)}
$$

In the case of contour parameterization through Bsplines control points, from Green's theorem in the plane it is easy to show that the area enclosed by a curve with parametrization $X(u)$ and $Y(u)$ is given by:

$$
A(t)=\int_{u_{\min }}^{u_{\max }} X(u ; t) \frac{d}{d u} Y(u ; t) d u .
$$

For a B-spline curve it can be shown that the area is given by:

$$
\begin{aligned}
A(t) & =\int_{u_{\min }}^{u_{\max }} \sum_{i} \sum_{j}\left(X_{i} Y_{j}\right) B_{i k} \frac{d}{d u} B_{j k} d u \\
& =\sum_{i} \sum_{j}\left(X_{i} Y_{j}\right) \int_{u_{\min }}^{u_{\max }} B_{i k} \frac{d}{d u} B_{j k} d u .
\end{aligned}
$$

In matrix notation one gets,

$$
A(t)=\mathbf{X}^{\prime}(t) S \mathbf{Y}(t)
$$

and its time derivative:

$$
\dot{A}(t)=\dot{\mathbf{X}}^{\prime}(t) S \mathbf{Y}(t)+\mathbf{X}^{\prime}(t) S \dot{\mathbf{Y}}(t)
$$

where $S=\left\{s_{i j}\right\}, s_{i j}=\int_{u_{\min }}^{u_{\max }} B_{i k} \frac{d}{d u} B_{j k} d u$. Note that both $A(t)$ and $\dot{A}(t)$ are multilinear function of the control points and their derivatives.

Notice that the entries of matrix $S$ in $A(t)$ and $\dot{A}(t)$ are integral parameters which can be computed quite inexpensively at any iteration of the set membership filter recursion, described in Section 2.3. In fact, $X_{i}, Y_{i}, \dot{X}_{i}, \dot{Y}_{i}$ are exactly the state variables of the contour dynamics model. Moreover, since the integral factors do not depend on time $t$, they can be computed off-line without affecting the computational burden of the tracking algorithm. Finally, notice that the integral nature of the parameters used to compute $\tau$ provides a natural noise filter, ensuring a reliable degree of stability of the recursive estimates of the time to contact.

The feasibility region $\mathcal{P}(k \mid k)$ associated with the estimate provided by the set membership filter can be employed to compute upper and lower bounds on the time to contact estimate, as it will be illustrated in Section 3 .

For the Q-parameterization, let us separate equation (5) as

$$
\left[\begin{array}{l}
\mathbf{X} \\
\mathbf{Y}
\end{array}\right]=\left[\begin{array}{l}
W_{x} \\
W_{y}
\end{array}\right] \mathbf{Q}+\left[\begin{array}{c}
\overline{\mathbf{X}} \\
\overline{\mathbf{Y}}
\end{array}\right]
$$

The area contained in the image contour and its derivative become

$$
\begin{gathered}
A(t)=\mathbf{Q}^{\prime} W_{x}^{\prime} S W_{y} \mathbf{Q}+\overline{\mathbf{Y}}^{\prime} S W_{x} \mathbf{Q}+\overline{\mathbf{X}}^{\prime} S W_{y} \mathbf{Q}+\overline{\mathbf{X}}^{\prime} S \overline{\mathbf{Y}} \\
\dot{A}(t)=\mathbf{Q}^{\prime}\left(W_{x}^{\prime} S W_{y}+W_{x} S W_{y}^{\prime}\right) \dot{\mathbf{Q}}+\overline{\mathbf{Y}}^{\prime} S W_{x} \dot{\mathbf{Q}}+\overline{\mathbf{X}}^{\prime} S W_{y} \dot{\mathbf{Q}} .
\end{gathered}
$$

Due to the particular structure of matrices $W_{x}$ and $W_{y}$, the diagonal elements of matrix $W_{x}^{\prime} S W_{y}$ are null. Hence also for the Q-parameterization case, $A(t)$ and $\dot{A}(t)$ are multilinear functions of the state vector $\left[\mathbf{Q}^{\prime} \dot{\mathbf{Q}}^{\prime}\right]$ components.

Observe that multilinearity will play a key role in computing the bounds on the time to contact estimate, cf. Section 3. 


\section{Exact error bounds on the time-to-contact estimate}

Let

$$
\lambda=\left[\mathbf{X}^{\prime}, \mathbf{Y}^{\prime}, \dot{\mathbf{X}}^{\prime}, \dot{\mathbf{Y}}^{\prime}\right]^{\prime}
$$

or

$$
\lambda=\left[\mathbf{Q}^{\prime}, \dot{\mathbf{Q}}^{\prime}\right]^{\prime}
$$

and let $\mathcal{B}$ be the estimated uncertainty box for the control points coordinates or for the affine transformation variables and their respective time derivatives. Following (22), this set can be written as

$$
\mathcal{B}=\left\{\lambda \in \mathbb{R}^{M}: \hat{\lambda}_{i}-d_{i} \leq \lambda_{i} \leq \hat{\lambda}_{i}+d_{i}, i=1, \ldots, M\right\}
$$

where $M=4 m$ for control points and $M=12$ for affine variables.

According to (23), the time to contact $\tau(\lambda)$ is given by

$$
\tau(\lambda)=n(\lambda) / d(\lambda)=2 A(t) / \dot{A}(t) .
$$

From $(24,25)$ and from $(27,28)$, it ensues that, for both the control points and the affine parameterization, the time to contact (29) is given by the ratio of two multilinear function in the respective state space variable $\lambda$.

Now, we want to compute the uncertainty interval $\left[\tau_{\min }, \tau_{\max }\right]$ on the time to contact, induced by uncertainty in the parameter vector $\lambda$, i.e. we want to solve the following constrained optimization problems

$$
\tau_{\text {min }}=\min _{\lambda \in \mathcal{B}} \tau(\lambda), \quad \tau_{\max }=\max _{\lambda \in \mathcal{B}} \tau(\lambda) .
$$

Notice that problems (30) are nonconvex, being the objective function the ratio of two multilinear functions of the independent variables. We show in the following that both problems (30) can be solved in a finite number of steps.

First, we provide a lemma which will be used later. Let us introduce an important extremal subset of $\mathcal{B}$, made of the vertices of the hyperrectangle $\mathcal{B}$

$$
\mathcal{B}_{v}= \begin{cases} & \lambda \in \mathcal{B}: \lambda_{i}=\hat{\lambda}_{i}+\alpha_{i} d_{i}, \\ & \left.\alpha_{i} \in\{-1,1\}, i=1, \ldots, M\right\} .\end{cases}
$$

Lemma 2. Let $f(\lambda): R^{M} \rightarrow R$ be a multilinear function of $\lambda$. Then

$$
\begin{aligned}
& \max _{\lambda \in \mathcal{B}} f(\lambda)=\max _{\lambda \in \mathcal{B}_{v}} f(\lambda), \\
& \min _{\lambda \in \mathcal{B}} f(\lambda)=\min _{\lambda \in \mathcal{B}_{v}} f(\lambda) .
\end{aligned}
$$

Now, we can state and prove the main result of this section.

Theorem 1. Let the time to contact be defined as in $(29,24,25)$ or as in $(29,27,28)$. Then

$$
\tau_{\min }=\min _{\lambda \in \mathcal{B}_{v}} \tau(\lambda), \quad \tau_{\max }=\max _{\lambda \in \mathcal{B}_{v}} \tau(\lambda) .
$$

Proof.

With reference to the definition of $\tau$ in (29), let us introduce the function

$$
F(\tau ; \lambda)=\tau d(\lambda)-n(\lambda) .
$$

For a fixed $\tau$, define the value set

$$
\mathcal{V}(\tau)=\{x \in \mathbb{R}: x=F(\tau ; \lambda), \text { for some } \lambda \in \mathcal{B}\} .
$$

The set $\mathcal{V}(\tau)$ is a segment of the real axis, whose left and right endpoints are

$$
E^{l}(\tau)=\min _{\lambda \in \mathcal{B}} F(\tau ; \lambda), E^{r}(\tau)=\max _{\lambda \in \mathcal{B}} F(\tau ; \lambda) .
$$

From the definition of $\tau$ in (29), it is easy to verify that $\emptyset \in \mathcal{V}(\tau)$, if and only if $\tau_{\min } \leq \tau \leq \tau_{\max }$. By a continuity argument, the minimum and maximum values of $\tau$ for which the equation

$$
F(\tau ; \lambda)=0
$$

admits a solution for some $\tilde{\lambda} \in \mathcal{B}$, are values of $\tau$ such that one of the endpoints of the value set $\mathcal{V}(\tau)$ coincides with the origin of the real axis, i.e.

$$
\begin{aligned}
& E^{r}\left(\tau_{\min }\right)=0 \text { or } E^{l}\left(\tau_{\min }\right)=0 \\
& E^{r}\left(\tau_{\max }\right)=0 \text { or } E^{l}\left(\tau_{\max }\right)=0 .
\end{aligned}
$$

Since the function $F(\tau ; \lambda)$ is multilinear in $\lambda$, by Lemma 2 it is concluded that for any fixed $\tau$, and hence also for $\tau=\tau_{\min }$ and $\tau=\tau_{\max }$, there exist $\lambda_{v}^{m}, \lambda_{v}^{M} \in \mathcal{B}_{v}$ such that

$$
\begin{aligned}
\lambda_{v}^{m} & =\arg \left\{\min _{\lambda \in \mathcal{B}} F(\tau ; \lambda)\right\}, \\
\lambda_{v}^{M} & =\arg \left\{\max _{\lambda \in \mathcal{B}} F(\tau ; \lambda)\right\} .
\end{aligned}
$$

This completes the proof.

Remark 1 Theorem 1 states that the evaluation of the bounds on $\tau$ can be made by simply considering a very special extremal subset of the entire parameter uncertainty set. In principle, this result reduces a nonconvex optimization problem to a finite time search algorithm.

Remark 2 The main difference between using the control points and the affine parameterization is that in the first approach, the number of vertices may be high, depending on the number of control points used to parameterize the contour of the considered image area. However, since for the time to contact problem the reference contour on the object is chosen by the operator, in practical applications it is always possible to use a limited number of control points.

In the second approach the dimension of the state vector does not depend on the number of control points and it is always equal to 12 . If the number of control points is greater than 3 , the time to contact estimation 
procedure becomes faster. Under the hypothesis of uniform relative motion along the optical axis, traslational parameters in (4) are identically zero and the dimension of the state vector $\lambda$. reduces to 8 , thus increasing the computational efficiency of the affine parameterization approach. However, it should be noted that the affine parameterization is less general than parameterization by control points, because it exactly applies only for moving planar rigid objects.

Remark 3 Note that, if the optical flow approach [12] is used, the parameter vector to be estimated is $\mu=$ $\left[u_{o}, v_{o}, u_{x}, v_{x}, u_{y}, v_{y}, \dot{u}_{o}\right]^{\prime} \in \mathbb{R}^{7}$ whose components are the coefficients of the first-order approximation of the optical flow (on the image plane). The time to contact is given by $\tau(\mu)=n(\mu) / d(\mu)$, where

$$
\begin{aligned}
n(\mu)= & \dot{u}_{0}+u_{0} u_{x}-u_{0} v_{y}+v_{0} v_{x}+v_{0} u_{y} \\
d(\mu)= & v_{y}\left(\dot{u}_{0}+u_{0} u_{x}-u_{0} v_{y}+v_{0} v_{x}+v_{0} u_{y}\right. \\
& \left.-\tilde{x} v_{y}^{2}+\tilde{x} u_{x} v_{y}+\tilde{y} v_{x} v_{y}+\tilde{y} u_{y} v_{y}\right) .
\end{aligned}
$$

In this case $n(\mu)$ is multilinear in $\mu$, while $d(\mu)$ is multilinear for any fixed value of $\mu_{6}=v_{y}$. As a consequence, for any fixed value of $\mu_{6}$, the time to contact is again the ratio of two multilinear functions and the computation of its uncertainty interval requires two one dimensional searches on a finite interval $[9,10]$.

\section{Conclusions}

In this paper the set-membership estimation theory has been applied to the contour tracking problem in dynamic vision. The aim was to estimate the time to contact with the differential invariant approach. Due to the nature of the set-membership filtering, exact error bounds on the time to contact were obtained as solutions of two non-convex optimization problems. From a computational point of view, it has been shown that such problems can be solved through finite time searches.

\section{References}

[1] R. Bartels, J. Beatty, and J. B. Barsky. An Introduction to Splines for Use in Compute Graphics and Geometric Modelling. Morgan Kaufmann, San Matteo, CA, 1987.

[2] D. P. Bertseikas and I. B. Rhodes. Recursive state estimation for a set-membership description of uncertainty. IEEE Transactions on Automatic Control, 16:117-128, 1971.

[3] A. Blake, R. Curwen, and A. Zisserman. A framework for spatio-temporal control in the tracking of visual contours. Int. Journal of Computer Vision, 11:127-145, 1993.
[4] Andrew Blake, Michael Isard, and David Raynard. Learning to track visual motion of contours. Artificial Intelligence, 78:101-133, 1995.

[5] F. L. Chernousko. Optimal guaranteed estimates of indeterminacies with the aid of ellipsoids. part i. Eng. Cybernetics, 18:1-9, 1980.

[6] L. Chisci, A. Garulli, and G. Zappa. Recursive state bounding by parallelotopes. Automatica, 32:10491055, 1996.

[7] R. Cipolla. Active Visual Inference of Surface Shape. Lecture Notes in Computer Science, 1016, Springer, 1996.

[8] M. A. Fischler and R. A. Elschlager. The representation and matching of pictorial structures. IEEE Trans. Computers, C-22(1), 1973.

[9] A. Garulli, D. Prattichizzo, and A. Vicino. Uncertainty interval evaluation for time to contact estimation problems. In Proc. 3rd IFAC Symp. on Intelligent Autonomous Vehicles, IAV'98, Spain 1988.

[10] A. Garulli, D. Prattichizzo, and A. Vicino. Set membership techniques for dynamic vision. Technical Report, Università di Siena, Italia 1988.

[11] A. B. Kurzhanski and V. M. Veliov. Modeling Techniques for Uncertain Systems. Birkhauser, Boston, 1994.

[12] F. G. Meyer. Time-to-collision from first-order models of the motion field. IEEE Transaction on Robotics and Automation, 10:792-798, 1994.

[13] M. Milanese, R. Tempo, and A. Vicino, editors. Robustness in Identification and Control. Plenum Press, New York, 1988.

[14] M. Milanese and A. Vicino. Optimal estimation theory for dynamic systems with set membership uncertainty: an overview. Automatica, 27(6):997-1009, 1991.

[15] R. C. Nelson and J. Aloimonos. Using flow field divergence for obstacle avoidance: towards qualitative vision. In Proc. 2nd Int. Conf. on Computer Vision, pages $188-196,1988$.

[16] F. C. Schweppe. Uncertain Dynamic Systems. Prentice Hall, Englewood Cliffs, NJ, 1973.

[17] M. Subbarao. Bounds on time-to-collision and rotational component from first order derivatives of image flow. CVGIP, 50:329-341, 1990 .

[18] S. Ullman and R. Basri. Recognition by linear combination of model. IEEE Trans. Pattern Analysis Machine Intelligence, 13(10):992-1006, 1991.

[19] A. Vicino and G. Zappa. Sequential approximation of feasible parameter sets for identification with set membership uncertainty. IEEE Transactions on Automatic Control, 41:774-785, 1996. 